\title{
UNIQUENESS OF MEROMORPHIC FUNCTIONS CONCERNING WEAKLY WEIGHTED-SHARING
}

\author{
ShanhUa Lin AND WeICHUAN LiN
}

\begin{abstract}
In this paper, we introduce the definition of weakly weighted-sharing which is between "CM" and "IM". Using the notion of weakly weighted-sharing, we study the uniqueness problems on meromorphic function and its $k$ th order derivative $f^{(k)}$ satisfying certain sharing set properties. As consequences, we are able to answer questions posed by Kit-wing $\mathrm{Yu}$, which were also studied by I. Lahiri and A. Sarkar, L. P. Liu and Y. X. Gu. Our results sharpen the above results.
\end{abstract}

\section{Introduction and main results}

In this paper, we shall use the standard notations in Nevanlinna's value distribution theory of meromorphic functions such as $T(r, f), N(r, f)$ and $m(r, f)$ (see W. K. Hayman [1] or L. Yang [2]). The notation $S(r, f)$ is defined to be any quantity satisfying $S(r, f)=o(T(r, f))$ as $r \rightarrow \infty$ possibly outside a set of $r$ of finite linear measure. A meromorphic function $a$ is called a small function with respect to $f$ provided that $T(r, a)=S(r, f)$. Denote $S(f)$ the set of all small functions of $f$.

For any two nonconstant meromorphic functions $f$ and $g$, and $a \in S(f)$, we say that $f$ and $g$ share $a \mathrm{IM}(\mathrm{CM})$ provided that $f-a$ and $g-a$ have the same zeros ignoring(counting) multiplicities. If $\frac{1}{f}$ and $\frac{1}{g}$ share 0 IM $(\mathrm{CM})$, we say that
$f$ and $g$ share $\infty$ IM $(\mathrm{CM})$.

Let $N_{E}(r, a)$ be the counting function of all common zeros of $f-a$ and $g-a$ with the same multiplicities, and $N_{0}(r, a)$ be the counting functions of all common zeros of $f-a$ and $g-a$ ignoring multiplicities. Denote by $\bar{N}_{E}(r, a)$ and $\bar{N}_{0}(r, a)$ the reduced counting functions of $f$ and $g$ corresponding to the counting functions $N_{E}(r, a)$ and $N_{0}(r, a)$, respectively. If

$$
\bar{N}\left(r, \frac{1}{f-a}\right)+\bar{N}\left(r, \frac{1}{g-a}\right)-2 \bar{N}_{E}(r, a)=S(r, f)+S(r, g),
$$

2000 Mathematics Subject Classification. 30D35, 30D45.

Key words. Weakly weighted sharing, uniqueness, meromorphic function.

Received August 30, 2005. 
then we say that $f$ and $g$ share $a$ "CM". If

$$
\bar{N}\left(r, \frac{1}{f-a}\right)+\bar{N}\left(r, \frac{1}{g-a}\right)-2 \bar{N}_{0}(r, a)=S(r, f)+S(r, g),
$$

then we say that $f$ and $g$ share $a$ "IM".

Definition 1 [3-4]. Let $k$ be a positive integer, and let $f$ be a meromorphic function and $a \in S(f)$.

(i) $\bar{N}_{k)}\left(r, \frac{1}{f-a}\right)$ denotes the counting function of those $a$-points of $f$ whose multiplicities are not greater than $k$, where each $a$-point is counted only once.

(ii) $\bar{N}_{(k}\left(r, \frac{1}{f-a}\right)$ denotes the counting function of those $a$-points of $f$ whose multiplicities are not less than $k$, where each $a$-point is counted only once.

(iii) $N_{p}\left(r, \frac{1}{f-a}\right)$ denotes the counting function of those $a$-points of $f$, where an $a$-point of $f$ with multiplicity $m$ counted $m$ times if $m \leq p$ and $p$ times if $m>p$.

Definition 2 [5]. We denote by $\delta_{p}(a, f)$ the quantity

$$
\delta_{p}(a, f)=1-\varlimsup_{r \rightarrow \infty} \frac{N_{p}\left(r, \frac{1}{f-a}\right)}{T(r, f)},
$$

where $p$ is a positive integer.

Clearly $\delta_{p}(a, f) \geq \delta(a, f)$.

In 2003, Kit-wing $\mathrm{Yu}$ [6] considered the uniqueness problem of an entire function or meromorphic function when it shares one small function with its derivative and proved the following results.

THEOREM A. Let $k \geq 1$. Let $f$ be a non-constant entire function, $a \in S(f)$ and $a \neq \equiv, \infty$. If $f, f^{(k)}$ share $a C M$ and $\delta(0, f)>\frac{3}{4}$, then $f \equiv f^{(k)}$.

THEOREM B. Let $k \geq 1$. Let $f$ be a non-constant non-entire meromorphic function, $a \in S(f)$ and $a \neq 0, \infty, f$ and $a$ do not have any common pole. If $f$, $f^{(k)}$ share a $C M$ and $4 \delta(0, f)+2(8+k) \Theta(\infty, f)>19+2 k$, then $f \equiv f^{(k)}$.

In the same paper, Kit-wing $\mathrm{Yu}$ posed the following open questions:

Question 1. Can a CM shared value be replaced by an IM shared value in Theorem A? 
QUESTION 2. Is the condition $\delta(0, f)>\frac{3}{4}$ sharp in Theorem A?

QUESTION 3. Is the condition $4 \delta(0, f)+2(8+k) \Theta(\infty, f)>19+2 k$ sharp in Theorem B?

Question 4. Can the condition " $f$ and $a$ do not have any common pole" be deleted in Theorem B?

In 2004, L. P. Liu and Y. X. Gu [7] applied a different method and obtained the following results.

THEOREM C. Let $f$ be a non-constant meromorphic function, $a \in S(f)$ and $a \not \equiv, \infty$. If $f, f^{(k)}$ share a $C M, f^{(k)}$ and a do not have any common pole of same multiplicity and $2 \delta(0, f)+4 \Theta(\infty, f)>5$, then $f \equiv f^{(k)}$.

THEOREM D. Let $f$ be a non-constant entire function, $a \in S(f)$ and $a \neq 0, \infty$. If $f, f^{(k)}$ share a $C M$ and $\delta(0, f)>\frac{1}{2}$, then $f \equiv f^{(k)}$.

In this paper, we introduce the definition of weakly weighted-sharing. By the new definition, we obtain uniqueness theorems which answer the questions posed by Kit-wing Yu. Moreover, our results improve Theorem A, B, C, D mentioned above.

Next, we introduce some notations for our definition.

Definition 3. Let $f$ and $g$ be two nonconstant meromorphic functions sharing $a$ "IM", for $a \in S(f) \cap S(g)$, and a positive integer $k$ or $\infty$.

(i) $\bar{N}_{k)}^{E}(r, a)$ denotes the counting function of those $a$-points of $f$ whose multiplicities are equal to the corresponding $a$-points of $g$, both of their multiplicities are not greater than $k$, where each $a$-point is counted only once.

(ii) $\bar{N}_{(k}^{o}(r, a)$ denotes the reduced counting function of those $a$-points of $f$ which are $a$-points of $g$, both of their multiplicities are not less than $k$, where each $a$-point is counted only once.

Definition 4. For $a \in S(f) \cap S(g)$, if $k$ is a positive integer or $\infty$, and

$$
\begin{gathered}
\bar{N}_{k)}\left(r, \frac{1}{f-a}\right)-\bar{N}_{k)}^{E}(r, a)=S(r, f), \quad \bar{N}_{k)}\left(r, \frac{1}{g-a}\right)-\bar{N}_{k)}^{E}(r, a)=S(r, g) ; \\
\bar{N}_{(k+1}\left(r, \frac{1}{f-a}\right)-\bar{N}_{(k+1}^{o}(r, a)=S(r, f), \quad \bar{N}_{(k+1}\left(r, \frac{1}{g-a}\right)-\bar{N}_{(k+1}^{o}(r, a)=S(r, g) .
\end{gathered}
$$

Or if $k=0$ and

$$
\bar{N}\left(r, \frac{1}{f-a}\right)-\bar{N}_{0}(r, a)=S(r, f), \quad \bar{N}\left(r, \frac{1}{g-a}\right)-\bar{N}_{0}(r, a)=S(r, g),
$$


then we say $f$ and $g$ weakly share $a$ with weight $k$. Here, we write $f, g$ share " $(a, k)$ " to mean that $f, g$ weakly share $a$ with weight $k$.

Obviously, if $f$ and $g$ share " $(a, k)$ ", then $f$ and $g$ share " $(a, p)$ " for any $p(0 \leq p \leq k)$. Also, we note that $f$ and $g$ share $a$ "IM" or "CM" if and only if $f$ and $g$ share " $(a, 0)$ " or " $(a, \infty)$ ", respectively.

Now, we state the main results of this paper.

THEOREM 1. Let $k \geq 1$ and $2 \leq m \leq \infty$. Let $f$ be a non-constant meromorphic function, $a \in S(f)$ and $a \neq 0, \infty$. If $f, f^{(k)}$ share " $(a, m)$ " and $2 \delta_{2+k}(0, f)+4 \Theta(\infty, f)>5$, then $f \equiv f^{(k)}$.

THEOREM 2. Let $k \geq 1$, and let $f$ be a non-constant meromorphic function, $a \in S(f)$ and $a \neq 0, \infty$. If $f, f^{(k)}$ share " $(a, 1)$ " and $\frac{5}{2} \delta_{2+k}(0, f)+\frac{k+9}{2} \Theta(\infty, f)$ $>\frac{k}{2}+6$, then $f \equiv f^{(k)}$.

THEOREM 3. Let $k \geq 1$, and let $f$ be a non-constant meromorphic function, $a \in S(f)$ and $a \neq \equiv, \infty$. If $f, f^{(k)}$ share $a$ "IM" and $5 \delta_{2+k}(0, f)+$ $(2 k+7) \Theta(\infty, f)>2 k+11$, then $f \equiv f^{(k)}$.

If $f$ is a nonconstant entire function, then $\Theta(\infty, f)=1$. So we have the following results.

COROllary 1. Let $k \geq 1$ and $2 \leq m \leq \infty$. Let $f$ be a non-constant entire function, $a \in S(f)$ and $a \neq 0, \infty$. If $f, f^{(k)}$ share " $(a, m)$ " and $\delta_{2+k}(0, f)>\frac{1}{2}$, then $f \equiv f^{(k)}$.

COROllary 2. Let $k \geq 1$, and let $f$ be a non-constant entire function, $a \in S(f)$ and $a \neq \equiv, \infty$. If $f$ and $f^{(k)}$ share " $(a, 1)$ " and $\delta_{2+k}(0, f)>\frac{3}{5}$, then $f \equiv f^{(k)}$.

COROllary 3. Let $k \geq 1$, and let $f$ be a non-constant entire function, $a \in S(f)$ and $a \neq 0, \infty$. If $f, f^{(k)}$ share $a$ "IM" and $\delta_{2+k}(0, f)>\frac{4}{5}$, then $f \equiv f^{(k)}$.

Remark 1: Theorem 1 and Corollary 1 improve Theorem A-D. Theorem 2 and Corollary 2 improve Theorem A, B. Theorem 3 and Corollary 3 answer question 1. Meanwhile, we give an affirmative answer to the forth question.

\section{Some lemmas}

LEMMA 1 [3]. Let $f$ be a nonconstant meromorphic function and let $k$ be a positive integer. Then 
(i) $N\left(r, \frac{1}{f^{(k)}}\right) \leq N\left(r, \frac{1}{f}\right)+k \bar{N}(r, f)+S(r, f)$.

(ii) $N\left(r, \frac{1}{f^{(k)}}\right) \leq T\left(r, f^{(k)}\right)-T(r, f)+N\left(r, \frac{1}{f}\right)+S(r, f)$.

Next, we introduce some notations for the following lemma.

When $f$ and $g$ share 1 "IM", $\bar{N}^{L}\left(r, \frac{1}{f-1}\right)$ denotes the counting function of the 1-points of $f$ whose multiplicities are greater than 1-points of $g$, where each zero is counted only once. Similarly, we have $\bar{N}^{L}\left(r, \frac{1}{g-1}\right) \cdot N_{11}\left(r, \frac{1}{f-1}\right)$ denotes the counting function of common simple 1-points of $f$ and $g$.

LEMMA 2. Let $f$ be a nonconstant meromorphic function and let $k$ be $a$ positive integer. Then

(i) $N_{2}\left(r, \frac{1}{f^{(k)}}\right) \leq N_{2+k}\left(r, \frac{1}{f}\right)+k \bar{N}(r, f)+S(r, f)$.

(ii) $N_{2}\left(r, \frac{1}{f^{(k)}}\right) \leq T\left(r, f^{(k)}\right)-T(r, f)+N_{2+k}\left(r, \frac{1}{f}\right)+S(r, f)$.

Proof. By Lemma 1(i), we have

$$
\begin{aligned}
N_{2}\left(r, \frac{1}{f^{(k)}}\right)+\sum_{p=3}^{\infty} \bar{N}_{(p}\left(r, \frac{1}{f^{(k)}}\right) \leq & N_{2+k}\left(r, \frac{1}{f}\right)+\sum_{p=3+k}^{\infty} \bar{N}_{(p}\left(r, \frac{1}{f}\right) \\
& +k \bar{N}(r, f)+S(r, f),
\end{aligned}
$$

i.e.

$$
\begin{aligned}
N_{2}\left(r, \frac{1}{f^{(k)}}\right) \leq & N_{2+k}\left(r, \frac{1}{f}\right)+\sum_{p=3+k}^{\infty} \bar{N}_{(p}\left(r, \frac{1}{f}\right)-\sum_{p=3}^{\infty} \bar{N}_{(p}\left(r, \frac{1}{f^{(k)}}\right) \\
& +k \bar{N}(r, f)+S(r, f) \\
\leq & N_{2+k}\left(r, \frac{1}{f}\right)+k \bar{N}(r, f)+S(r, f) .
\end{aligned}
$$

(ii) can be followed by using part (i) directly.

Lemma 3. Let $m$ be a nonnegative integer or $\infty$. Let $F$ and $G$ be two nonconstant meromorphic functions, and $F, G$ share " $(1, m)$ ". Let

$$
H=\left(\frac{F^{\prime \prime}}{F^{\prime}}-2 \frac{F^{\prime}}{F-1}\right)-\left(\frac{G^{\prime \prime}}{G^{\prime}}-2 \frac{G^{\prime}}{G-1}\right) .
$$

If $H \not \equiv 0$, then 
(i) If $2 \leq m \leq \infty$, then

$T(r, F) \leq N_{2}(r, F)+N_{2}\left(r, \frac{1}{F}\right)+N_{2}(r, G)+N_{2}\left(r, \frac{1}{G}\right)+S(r, F)+S(r, G)$.

(ii) If $m=1$, then

$$
\begin{aligned}
T(r, F) \leq & N_{2}(r, F)+N_{2}\left(r, \frac{1}{F}\right)+N_{2}(r, G)+N_{2}\left(r, \frac{1}{G}\right)+\bar{N}^{L}\left(r, \frac{1}{F-1}\right) \\
& +S(r, F)+S(r, G) .
\end{aligned}
$$

(iii) If $m=0$, then

$$
\begin{aligned}
T(r, F) \leq & N_{2}(r, F)+N_{2}\left(r, \frac{1}{F}\right)+N_{2}(r, G)+N_{2}\left(r, \frac{1}{G}\right)+2 \bar{N}^{L}\left(r, \frac{1}{F-1}\right) \\
& +\bar{N}^{L}\left(r, \frac{1}{G-1}\right)+S(r, F)+S(r, G) .
\end{aligned}
$$

The same inequalities holds for $T(r, G)$. have

Proof. (i) If $2 \leq m \leq \infty$, then by the Second Fundamental Theorem, we

$$
T(r, F) \leq \bar{N}(r, F)+\bar{N}\left(r, \frac{1}{F}\right)+\bar{N}\left(r, \frac{1}{F-1}\right)-N_{0}\left(r, \frac{1}{F^{\prime}}\right)+S(r, F),
$$

where $N_{0}\left(r, \frac{1}{F^{\prime}}\right)$ is the counting function in $|z|<r$ of the zeros of $F^{\prime}$ that are not the zeros of $F$ and $F-1 . \quad N_{0}\left(r, \frac{1}{G^{\prime}}\right)$ can be defined similarly.

By a simple calculation, any pole of $F$ is not a pole of $\frac{F^{\prime \prime}}{F^{\prime}}-2 \frac{F^{\prime}}{F-1}$, any pole of $G$ is not a pole of $\frac{G^{\prime \prime}}{G^{\prime}}-2 \frac{G^{\prime}}{G-1}$. Furthermore, let $z_{1}$ be a common zero of $F-1$ and $G-1$ with multiplicity $t$, where $1 \leq t \leq 2$. We know that $H$ is analytic at $z_{1}$. Therefore, by $H \not \equiv 0$, we have

$$
N_{1)}\left(r, \frac{1}{F-1}\right) \leq \bar{N}\left(r, \frac{1}{H}\right)+S(r, F)+S(r, G) \leq T(r, H)+S(r, F)+S(r, G) .
$$

Note that $m(r, H)=S(r, F)+S(r, G)$ and

$$
\begin{aligned}
N(r, H) \leq & \bar{N}_{(2}(r, F)+\bar{N}_{(2}(r, G)+\bar{N}_{(2}\left(r, \frac{1}{F}\right)+\bar{N}_{(2}\left(r, \frac{1}{G}\right) \\
& +N_{0}\left(r, \frac{1}{F^{\prime}}\right)+N_{0}\left(r, \frac{1}{G^{\prime}}\right)+\bar{N}^{L}\left(r, \frac{1}{F-1}\right) \\
& +\bar{N}^{L}\left(r, \frac{1}{G-1}\right)+S(r, F)+S(r, G)
\end{aligned}
$$


By (2.2), we have

$$
\begin{aligned}
N_{1)}\left(r, \frac{1}{F-1}\right) \leq & \bar{N}_{(2}(r, F)+\bar{N}_{(2}(r, G)+\bar{N}_{(2}\left(r, \frac{1}{F}\right)+\bar{N}_{(2}\left(r, \frac{1}{G}\right) \\
& +N_{0}\left(r, \frac{1}{F^{\prime}}\right)+N_{0}\left(r, \frac{1}{G^{\prime}}\right)+\bar{N}^{L}\left(r, \frac{1}{F-1}\right) \\
& +\bar{N}^{L}\left(r, \frac{1}{G-1}\right)+S(r, F)+S(r, G) .
\end{aligned}
$$

Since $F$ and $G$ share " $(1, m)$ ", we have

$$
\begin{aligned}
& \bar{N}_{(2}\left(r, \frac{1}{G-1}\right)+\bar{N}^{L}\left(r, \frac{1}{F-1}\right)+\bar{N}^{L}\left(r, \frac{1}{F-1}\right) \\
& \quad+N_{0}\left(r, \frac{1}{G^{\prime}}\right)+N\left(r, \frac{1}{G}\right)-\bar{N}\left(r, \frac{1}{G}\right) \leq N\left(r, \frac{1}{G^{\prime}}\right) .
\end{aligned}
$$

It follows from Lemma 1 that

$$
\begin{aligned}
& \bar{N}_{(2}\left(r, \frac{1}{G-1}\right)+\bar{N}^{L}\left(r, \frac{1}{F-1}\right)+\bar{N}^{L}\left(r, \frac{1}{G-1}\right)+N_{0}\left(r, \frac{1}{G^{\prime}}\right) \\
& \quad \leq \bar{N}\left(r, \frac{1}{G}\right)+\bar{N}(r, G)+S(r, G) .
\end{aligned}
$$

In addition, we have

$$
\begin{aligned}
\bar{N}\left(r, \frac{1}{F-1}\right) & =N_{1)}\left(r, \frac{1}{F-1}\right)+\bar{N}_{(2}\left(r, \frac{1}{F-1}\right) \\
& =N_{1)}\left(r, \frac{1}{F-1}\right)+\bar{N}_{(2}\left(r, \frac{1}{G-1}\right) .
\end{aligned}
$$

Combining (2.1), (2.3), (2.4) and (2.5), we obtain

$$
T(r, F) \leq N_{2}(r, F)+N_{2}\left(r, \frac{1}{F}\right)+N_{2}(r, G)+N_{2}\left(r, \frac{1}{G}\right)+S(r, F)+S(r, G) .
$$

(ii) If $m=1$, then (2.4) is replaced by

$$
\bar{N}_{(2}\left(r, \frac{1}{G-1}\right)+\bar{N}^{L}\left(r, \frac{1}{G-1}\right)+N_{0}\left(r, \frac{1}{G^{\prime}}\right) \leq \bar{N}\left(r, \frac{1}{G}\right)+\bar{N}(r, G)+S(r, G) .
$$

Similar to the arguments in (i), we see that (ii) holds.

(iii) If $m=0$, then by a simple calculation, any common simple zero of $F-1$ and $G-1$ is zero of $H$. Therefore, by $H \not \equiv 0$, we have

$$
N_{11}\left(r, \frac{1}{F-1}\right) \leq \bar{N}\left(r, \frac{1}{H}\right)+S(r, F)+S(r, G) \leq T(r, H)+S(r, F)+S(r, G) .
$$


Thus

$$
\begin{aligned}
N_{11}\left(r, \frac{1}{F-1}\right) \leq & \bar{N}_{(2}(r, F)+\bar{N}_{(2}(r, G)+\bar{N}_{(2}\left(r, \frac{1}{F}\right)+\bar{N}_{(2}\left(r, \frac{1}{G}\right) \\
& +N_{0}\left(r, \frac{1}{F^{\prime}}\right)+N_{0}\left(r, \frac{1}{G^{\prime}}\right)+\bar{N}^{L}\left(r, \frac{1}{F-1}\right) \\
& +\bar{N}^{L}\left(r, \frac{1}{G-1}\right)+S(r, F)+S(r, G) .
\end{aligned}
$$

By the Second Fundament Theorem, we have

$$
\begin{aligned}
T(r, F)+T(r, G) \leq & \bar{N}(r, F)+\bar{N}\left(r, \frac{1}{F}\right)+\bar{N}\left(r, \frac{1}{F-1}\right)-N_{0}\left(r, \frac{1}{F^{\prime}}\right) \\
& +\bar{N}(r, G)+\bar{N}\left(r, \frac{1}{G}\right)+\bar{N}\left(r, \frac{1}{G-1}\right)-N_{0}\left(r, \frac{1}{G^{\prime}}\right) \\
& +S(r, F)+S(r, G),
\end{aligned}
$$

In addition, we have

$$
\begin{aligned}
\bar{N}\left(r, \frac{1}{F-1}\right)+\bar{N}\left(r, \frac{1}{G-1}\right)= & 2 \bar{N}\left(r, \frac{1}{G-1}\right)+S(r, F)+S(r, G) \\
\leq & N_{11}\left(r, \frac{1}{F-1}\right)+\bar{N}^{L}\left(r, \frac{1}{F-1}\right)+N\left(r, \frac{1}{G-1}\right) \\
& +S(r, F)+S(r, G) \\
\leq & N_{11}\left(r, \frac{1}{F-1}\right)+\bar{N}^{L}\left(r, \frac{1}{F-1}\right)+T(r, G) \\
& +S(r, F)+S(r, G) .
\end{aligned}
$$

Combining (2.6), (2.7) and (2.8), we obtain

$$
\begin{aligned}
T(r, F) \leq & N_{2}(r, F)+N_{2}\left(r, \frac{1}{F}\right)+N_{2}(r, G)+N_{2}\left(r, \frac{1}{G}\right)+2 \bar{N}^{L}\left(r, \frac{1}{F-1}\right) \\
& +\bar{N}^{L}\left(r, \frac{1}{G-1}\right)+S(r, F)+S(r, G) .
\end{aligned}
$$

LEMma 4 [8]. Let $f$ be a transcendental meromorphic function and $\alpha(\not \equiv 0, \infty)$ be a meromorphic function such that $T(r, \alpha)=S(r, f)$. Let $b$ and $c$ are any two finite nonzero distinct complex numbers. If $\psi=\alpha(f)^{n}\left(f^{(k)}\right)^{p}$, where $n(\geq 0), p(\geq 1)$ and $k(\geq 1)$ are integers, then

$$
\begin{aligned}
(p+n) T(r, f) \leq & (p+n) N\left(r, \frac{1}{f}\right)+N\left(r, \frac{1}{\psi-b}\right)+N\left(r, \frac{1}{\psi-c}\right)-N(r, f) \\
& -N\left(r, \frac{1}{\psi^{\prime}}\right)+S(r, f) .
\end{aligned}
$$




\section{Proofs of main theorems}

Proof of Theorem 1.

Let

$$
F=\frac{f}{a}, \quad G=\frac{f^{(k)}}{a} .
$$

Then it is easy to verify $F$ and $G$ share " $(1, m)$ ".

Let $H$ be defined as in Lemma 3. Suppose that $H \not \equiv 0$. It follows from Lemma 3 that

$$
T(r, G) \leq N_{2}(r, F)+N_{2}\left(r, \frac{1}{F}\right)+N_{2}(r, G)+N_{2}\left(r, \frac{1}{G}\right)+S(r, F)+S(r, G) .
$$

Using Lemma 2, we have

$$
\begin{aligned}
T\left(r, f^{(k)}\right) & \leq N_{2}(r, f)+N_{2}\left(r, \frac{1}{f}\right)+N_{2}\left(r, f^{(k)}\right)+N_{2}\left(r, \frac{1}{f^{(k)}}\right)+S(r, f) \\
& \leq N_{2+k}\left(r, \frac{1}{f}\right)+T\left(r, f^{(k)}\right)-T(r, f)+N_{2+k}\left(r, \frac{1}{f}\right)+4 \bar{N}(r, f)+S(r, f),
\end{aligned}
$$

i.e.

$$
T(r, f) \leq 2 N_{2+k}\left(r, \frac{1}{f}\right)+4 \bar{N}(r, f)+S(r, f) .
$$

It follows that $2 \delta_{2+k}(0, f)+4 \Theta(\infty, f) \leq 5$, which contradicts $2 \delta_{2+k}(0, f)+$ $4 \Theta(\infty, f)>5$. Therefore $H \equiv 0$. That is

$$
\frac{F^{\prime \prime}}{F^{\prime}}-2 \frac{F^{\prime}}{F-1} \equiv \frac{G^{\prime \prime}}{G^{\prime}}-2 \frac{G^{\prime}}{G-1} .
$$

It follows that

$$
\frac{1}{F-1}=\frac{A}{G-1}+B
$$

where $A(\neq 0)$ and $B$ are constants. Therefore,

$$
F=\frac{(B+1) G+(A-B-1)}{B G+(A-B)} .
$$

and

$$
T(r, F)=T(r, G)+S(r, f) .
$$

Now we distinguish the following two cases.

CASE 1. Suppose that $B \neq-1,0$. If $A$

If $A-B-1 \neq 0$, then from (3.2), we have $\bar{N}\left(r, \frac{1}{G+\frac{A-B-1}{B+1}}\right)=$
$\left(\frac{1}{F}\right)$. 
By the Second Fundamental Theorem, we have $T(r, G)<\bar{N}(r, G)+$ $\begin{aligned} \bar{N}\left(r, \frac{1}{G}\right)+ & \bar{N}\left(r, \frac{1}{G+\frac{A-B-1}{B+1}}\right)+S(r, G), \text { i.e. } \\ T\left(r, f^{(k)}\right) & <\bar{N}(r, f)+\bar{N}\left(r, \frac{1}{f^{(k)}}\right)+\bar{N}\left(r, \frac{1}{f}\right)+S(r, f) \\ & \leq \bar{N}(r, f)+T\left(r, f^{(k)}\right)-T(r, f)+N_{2+k}\left(r, \frac{1}{f}\right)+\bar{N}\left(r, \frac{1}{f}\right)+S(r, f),\end{aligned}$

and so

$$
T(r, f)<\bar{N}(r, f)+2 N_{2+k}\left(r, \frac{1}{f}\right)+S(r, f) .
$$

It follows that $2 \delta_{2+k}(0, f)+\Theta(\infty, f) \leq 2$, which contradicts $2 \delta_{2+k}(0, f)+$ $4 \Theta(\infty, f)>5 . \quad$ Therefore, $A-B-1=0 . \quad$ From (3.2), we obtain $\bar{N}\left(r, \frac{1}{G+\frac{1}{B}}\right)$
$=\bar{N}(r, F)$.

Similar to the arguments in the above, we also have a contradiction.

CASE 2. Suppose that $B=-1$.

If $A+1 \neq 0$. Then from (3.2), we have $\bar{N}\left(r, \frac{1}{G-(A+1)}\right)=\bar{N}(r, F)$.

Similar to the arguments in Case 1 , we can get a contradiction. Therefore, $A+1=0$, then from (3.2), we have $F G \equiv 1$. From (3.1), we have

$$
f f^{(k)} \equiv a^{2} .
$$

In the following, we distinguish two subcases.

a) If $f$ is a rational function, then $a$ becomes a nonzero constant. So from (3.1), we see that $f$ has no zero and pole. Since $f$ is nonconstant, this is a contradiction.

b) If $f$ is transcendental then by Lemma 4, we get in view of (3.1)

$$
\begin{aligned}
2 T(r, f) & \leq 2 N\left(r, \frac{1}{f}\right)+2 T\left(r, f f^{(k)}\right)+S(r, f) \\
& \leq 2 N\left(r, \frac{1}{f}\right)+S(r, f) \\
& \leq 2 N\left(r, \frac{1}{a^{2}}\right)+S(r, f)=S(r, f),
\end{aligned}
$$

This is a contradiction.

CASE 3. Suppose that $B=0$.
If $A-1 \neq 0$, then from $(3.2)$, we have $\bar{N}\left(r, \frac{1}{G+(A-1)}\right)=\bar{N}\left(r, \frac{1}{F}\right)$. 
Similar to the arguments in case 1 , we also have a contradiction. Therefore, $A-1=0$. From (3.2), we have $F \equiv G$, this implies $f \equiv f^{(k)}$.

This completes the proof of the Theorem 1.

Proof of Theorem 2. With the same notations, since $f, f^{(k)}$ share " $(a, 1)$ ", we obtain that $F, G$ share " $(1,1)$ ".

Let $H$ be defined as in Lemma 3. Suppose that $H \not \equiv 0$. It follows from Lemma 3 that

$$
\begin{aligned}
T(r, G) \leq & N_{2}(r, F)+N_{2}\left(r, \frac{1}{F}\right)+N_{2}(r, G)+N_{2}\left(r, \frac{1}{G}\right)+\bar{N}^{L}\left(r, \frac{1}{G-1}\right) \\
& +S(r, F)+S(r, G) .
\end{aligned}
$$

Since

$$
\begin{aligned}
\bar{N}^{L}\left(r, \frac{1}{G-1}\right) & \leq \frac{1}{2} N\left(r, \frac{G}{G^{\prime}}\right) \\
& \leq \frac{1}{2} N\left(r, \frac{G^{\prime}}{G}\right)+S(r, f) \\
& \leq \frac{1}{2} \bar{N}(r, G)+\frac{1}{2} \bar{N}\left(r, \frac{1}{G}\right)+S(r, f) \\
& \leq \frac{1}{2} \bar{N}(r, f)+\frac{1}{2} \bar{N}\left(r, \frac{1}{f^{(k)}}\right)+S(r, f) .
\end{aligned}
$$

Using Lemma 2, we have

$$
\begin{aligned}
T\left(r, f^{(k)}\right) \leq & N_{2}(r, f)+N_{2}\left(r, \frac{1}{f}\right)+N_{2}\left(r, f^{(k)}\right)+N_{2}\left(r, \frac{1}{f^{(k)}}\right)+\frac{1}{2} \bar{N}(r, f) \\
& +\frac{1}{2} \bar{N}\left(r, \frac{1}{f^{(k)}}\right)+S(r, f) \\
\leq & N_{2+k}\left(r, \frac{1}{f}\right)+T\left(r, f^{(k)}\right)-T(r, f)+N_{2+k}\left(r, \frac{1}{f}\right)+\frac{1}{2} N_{2+k}\left(r, \frac{1}{f}\right) \\
& +\frac{k+9}{2} \bar{N}(r, f)+S(r, f) .
\end{aligned}
$$

i.e.

$$
T(r, f) \leq \frac{5}{2} N_{2+k}\left(r, \frac{1}{f}\right)+\frac{k+9}{2} \bar{N}(r, f)+S(r, f),
$$

It follows that $\frac{5}{2} \delta_{2+k}(0, f)+\frac{k+9}{2} \Theta(\infty, f) \leq \frac{k}{2}+6$, which contradicts $\frac{5}{2} \delta(0, f)+\frac{k+9}{2} \Theta(\infty, f)>\frac{k}{2}+6$.

Similar to the arguments in Theorem 1, we see that Theorem 2 holds. 
Proof of Theorem 3. Using Lemma 3(iii), note that

$$
\bar{N}^{L}\left(r, \frac{1}{F-1}\right) \leq N\left(r, \frac{F}{F^{\prime}}\right) \leq \bar{N}\left(r, \frac{1}{f}\right)+\bar{N}(r, f)+S(r, f),
$$

and

$$
\begin{aligned}
\bar{N}^{L}\left(r, \frac{1}{G-1}\right) & <\bar{N}\left(r, \frac{1}{f^{(k)}}\right)+\bar{N}(r, f)+S(r, f) \\
& <N_{2+k}\left(r, \frac{1}{f}\right)+(k+1) \bar{N}(r, f)+S(r, f) .
\end{aligned}
$$

Similar to the arguments in Theorem 1, we see that Theorem 3 holds.

\section{Acknowledgment}

The second author of the paper supported by the JSPS Post Fellowship Program, the Youth Science Technology Foundation of Fujian Province (140101). The both authors was supported in part by the Education Committee Foundation of Fujian Province (JA03174).

The authors would like to thank Professor Hongxun Yi and Professor Seiki Mori for their valuable conversation during the preparation of this project.

\section{REFERENCES}

[1] W. K. Hayman, Meromorphic functions, Clarendon Press, Oxford, 1964.

[2] L. YANG, Value distribution theory, Springer-Verlag, Berlin, 1993.

[3] H. X. Yi AND C. C. YANG, Uniqueness theory of meromorphic functions (in Chinese), Science Press, Beijing, 1995.

[4] H. X. YI, On characteristic function of a meromorphic function and its derivative, Indian $\mathbf{J}$. Math. 33 (1991), 119-133.

[5] I. LAHIRI AND A. SARKar, Uniqueness of a meromorphic function and its derivative, J. Inequal. Pure and Appl. Math. 5 (2004), 1-9.

[6] Kit-Wing YU, On entire and meromorphic functions that share small functions with their derivatives, J. Inequal. Pure and Appl. Math. 4 (2003), 1-7.

[7] L. P. LIU AND Y. X. Gu, Uniqueness of meromorphic functions that share one small function with their derivatives, Kodai. Math. J. 27 (2004), 272-279.

[8] I. LAhIRI AND S. Dewan, Inequealities arising out of the value distribution of a differential monomial, J. Inequal. Pure Appl. Math. 4 (2003), Art. 27.

\section{Department of Mathematics \\ FUJIAN NORMAL UNIVERSITY \\ FUZHOU 350007 \\ P. R. CHINA}

Department of Mathematics

FuJIAN NORMAL UNIVERSITY

FUZHOU 350007

P. R. CHINA

E-mail: sxlwc936@fjnu.edu.cn

Current Address:

Department of Mathematics

SHANTOU UNIVERSITY

515063

P. R. CHINA 